\title{
Regional Anatomical Nomenclature and Arthroscopic Terminology in Human Temporomandibular Joints
}

\author{
By \\ Ken-ICHIRo MURAKAMI and KaZUMASA HOSHINO \\ Departments of Anatomy, and Oral and Maxillofacial Surgery, \\ Faculty of Medicine, Kyoto University, Kyoto 606, Japan \\ - Received for Publication, December 1, 1981-
}

\begin{abstract}
Key Words: Temporomandibular joint, Regional anatomical nomenclature, Arthroscopic anatomy.

Summary: Regional anatomical and arthroscopic features studied in 20 temporomandibular joints of $\mathbf{1 9}$ human cadavers were described. For descriptive purposes, clinically useful arthroscopic terminology and regional anatomical nomenclature in human temporomandibular joints were proposed in this paper.
\end{abstract}

Arthroscopic diagnosis is often desirable for certain diseses of the temporomandibular joint (hereafter referred to as TMJ), but so far arthroscopy of this joint has not been commonly performed.

Arthroscopy was originally attempted by Takagi (1918) and his cumulative results were reported in 1939 (Takagi). Basic studies for arthrocopy were undertaken in human cadavers earlier by Burman (1931) and later by Iino (1939), but these investigators did not include the TMJ in their studies. Arthroscopy of the TMJ was apparently first attempted by Ohnishi (1975) using the Watanabe No. 24 arthroscope (Olympus Selfoscope) which was developed in 1970 by Watanabe. A few studies on arthrascopy and/or arthroscopic anatomical features have been reported in Japan.

In order to describe arthroscopic findings of the TMJ, it became necessary to establish clinically usable and meaningful arthroscopic terminology compatible with their corresponding regional anatomical nomenclature. However, the current anatomical nomenclature (Nomina Anatomica, 1977) is insufficient to cover all the nomenclature necessary for this purpose. Moreover, no detailed studies of intra-articular features of the TMJ have apparently been made either gross anatomically or arthroscopically in human. Therefore, the present study using human TMJs was undertaken and gross anatomical nomenclature and arthroscopic clinical terminology in the TMJ was proposed in this paper.

\section{Materials and methods}

Twenty TMJs examined in the present study were removed en bloc from 19 cadavers, ranging in age from 23 to 94 years (71.9 years of age on an average). Although these cadvers had been preserved by an improved embalming procedure (Bradbury and Hoshino, 1978), all 
TMJs examined were again immersed in $0.7 \%$ formalin solution for 48 hours for softening in order to increase their movability. Arthroscopic investigations were performed using the Watanabe No. 24 arthroscope (Olympus Selfocope) in eight TMJs followed by gross anatomical dissection. Gross anatomical investigations were made in $16 \mathrm{TMJs}$ altogether and the remaining four TMJs were sliced under frozen condition 48 hours after being kept at $-20^{\circ} \mathrm{C}$ in the deep freezer (National NR-308FC). Two TMJs were sliced at $5 \mathrm{~mm}$ thick sagittaly and other two coronally with a bone cutter (Maruto Power Cutter MC-305) into several slices.

Gross anatomical dissections of the TMJ were performed from the outside inward toward various intra-articular spaces via four different approaches. Macroscopic observations of the extraand intra-articular structures were pursued, after dissected specimens were immersed in water in order to make the appearance of soft tissues such as the synovial membrane as close to the arthroscopic appearance as possible. Out of 16 TMJs, 10 joints were approached anteriorly, and two cases each in the remaining 6 TMJs were examined via lateral, medial, and posterior approaches, respectively, in order to minimize undesirable displacement of the articular structures from their in situ locations.

Photographs were taken during arthroscopic investigations and also gross anatomical observations.

\section{Results}

I. Gross anatomical features and their nomenclature :

Macroscopic observations of the TMJ and its relations to the adjacent structures were undertaken in gross-anatomically dissected specimens and also in frozen sectioned slices. Regional anatomical nomenclature for gross anatomical features of the TMJ and its adjacent structures were selected in consideration of arthroscopic anatomical features. When appropriate nomenclature for certain structures is presently not available from Nomina Anatomica, new designation for these structures are propsed.

1) General features of the TMJ:

The TMJ is a synovial joint between the temporal bone and the mandible, and is functionally responsible as a craniomandibular articulation for the jaw movement including the occlusion (Figs. 1, 2).

2) Articular capsule:

Theoretically the articular capsule of the TMJ may be described to be a loose and thin membrane surrounding the TMJ, and is attached above to the temporal bone around the circumferences of the mandibular fossa and the articular tubercle, and below to the mandibular neck. However, as will be described later, some portions of the articular capsule are not identifiable. The lateral aspect of the articular capsule of the TMJ is reinforced by the lateral ligament (commonly known as the temporomandibular ligament) which is a broad, flat, and thin but very strong ligamentous band attaching above to the lower border of the zygoma and the articular tubercle and running backward toward the lateral and posterior aspects of the mandibular neck. The sphenomandibular ligament is located medial to the articular capsule of the TMJ, but is separated from the latter by the intervening structures. The sphenomandibular ligament and the stylomandibular ligament, both of which are classified as the accessory ligaments, could support the movement of the TMJ but do not reinforce the articular capsule. The lateral pterygoid muscle is fused with the outer surface of the TMJ anteriorly and anteromedially (Figs. 1,2). thus as will be mentioned in detail later, 
there is no membranous structure identifiable as the articular capsule in these areas. Posteriorly, the articular capsule is mostly fused with the retrodiscal fat pad and becomes unidentifiable as a distinct membranous structure (Fig. 1).

3) Articular surfaces:

The articular surfaces of the TMJ cover the temporal bone, the mandible and the articular disk. The articular surface of the temporal bone consists of the surfaces of the mandibular fossa and the articular tubercle (Fig. 1). The articular surface of the mandible is the surface of the mandibular head whose long axis is directed medialward and slightly backward (Fig. 3). These bony surfaces are covered by the fibrocartilage. The articular surface of the articular disk appears to be not covered by the cartilage. The synovial membrane lines the inner surface the articullar cavity other than the articular surfaces.

4) Articular disk:

The space between the articular surfaces of the temporal bone and the mandibular head is divided by the intervening articular disk into the superior articular cavity and the inferior articular cavity (Figs. 1, 2). Only in pathological conditions such as menisectomy or perforation of the articular disk, these two articular cavities communicate freely each other. In normal condition, each of them functions separately and the superior articular cavity serves for the gliding joint movement and the inferior one for the hinge joint movement. In this sense, the TMJ may be interpreted to consist of two independent joints in a single cranio-mandibular articulation.

The upper and lower surfaces of the dome-shaped articular disk form to become congruent with the contour of the temporal and mandibular articular surfaces. At its sagittal section, the upper surface of the articular disk shows the contour resmbling a lying sigmoid shape, and its posterior highest portion is designated as the discal eminece, and the anterior lower portion is designated as a fiat portion although it has slight sloping configuration (Fig. 1).

The lateral pterygoid muscle consists of the smaller upper portion and the larger lower portion. The lower portion inserts into the pterygoid fovea of the mandibular neck. The upper portion arising from the infratemporal surface of the greater wing of the sphenoid bone inserts into the anterior and anteromedial aspects of the TMJ and the inserting muscle fibers are interwoven with the fibrous tissues of the anterior portion of the articular disk (Figs. 1, 2, 3). The upper portion of the lateral pterygoid muscle has been known as the sphenomeniscus portion.

The lower surface of the articular disk is concave so that it may fit with the convex articular suface of the mandibular head. The concavity underneath the articular disk is designated as the discal fossa (Figs. 1, 3). At both sides of the discal fossa, the lateral and medial portions of the articuar disk extend downward close to the mandibular head and firmly attach to the lateral pole and the medial pole of the mandibular head, respectively (Fig. 2); thus the articular disk moves together with the forward and backward movements of the mandibular head. The articular disk blends posteriorly with the retrodiscal fat pad (Fig. 1). The surfaces of the articular disk other than its upper and lower articular surfaces are lined by the synovial membrane, which also lines their adjacent intra-articular portions of the lateral pterygoid muscle and the retrodiscal fat pad. The articular disk, at its sagittal section, is thickest posteriorly, thinnest at the intermediate portion which is sandwitched between the mandibular head 
and the transitional area of the temporal bone from the posterior aspect of the articular tubercle toward the anterior slope of the mandibular fossa. The anterior portion of the articular disk is thicker than the intermediate portion (Fig. 1). At the coronal section through the discal eminece, the articular disk is thickest at its central portion and tapers bilaterally (Fig. 2).

5) Articular cavities :

a) Superior articular cavity:

This is the temporo-discal interspace. The superior articular cavity is bounded above by the continuous articular surface covering the articular tubercle and the mandibular fossa, below by the articular suface on the upper surface of the articular disk, and elsewhere by the synovial membrane (Figs. 1, 4).

b) Inferior articular cavity:

This is the condylo-discal interspace. The inferior articular cavity is bounded above by the articular surface on the undersurface of the articular disk, below by the articular surface of the mandibular head, and elsewhere by the synovial membrane (Figs. 1, 3).

II. Arthroscopic anatomical features and their clinical terminology:

Prior to arthroscopic investigations, certain amounts of normal saline solution must be injected into the articular cavities with some pressure in order to widen the intra-articular spaces. This unavoidable procedure makes anatomical relations somewhat unnatural. This must be kept in mind iwhen arthroscopic features are interpreted.

As described in the preceding section, the 'TMJ may be subdivided into two functionally independent joints, each on which has its own articular cavity. Due to the presence of the intervening articular disk, these two articular cavities are rather complicated in these configurations for arthroscopic descriptions. In both cavities several intraarticular spaces may be subdivided; namely, four spaces in the superior articular cavity and three spaces in the inferior articular cavity.

1) Superior articular cavity:

The anterior and posterior portions of the superior articular cavity are surrounded only by the syno- vial membrane, and form the upper anterior synovial pouch and the upper posterier synovial pouch,respectively. The space between these synovial pouches is the intermediate space of the superior articular cavity, where the temporal articular surface articulates with the discal articular surface.

The temporal articular surface is covered by the fibrocatilage over the articular tubercle and the mandibular fossa. At biting position, the articular surface of the mandibular fossa makes contact with the upper articular surface of the articular disk. These articular surfaces, however, form interspace at various opening positions of the lower jaw. This interspace is designated as the higher intermediate space (Fig. 5).

When the lower jaw is protruded, both the articular disk and the mandibular head move together forward untill the upper discal articular surface makes contact with the temporal articular surface at its transitional area from the anterior slope of the mandibular fossa toward the posterior slope of the articular tubercle. When the lower jaw is retracted from its protrusive position, an interspace is then formed between these articular surfaces. This interspace is designated as the lower intermediate space (Fig. 6). When the lower intermediate space is closed by protrusion of the lower jaw, the higher intemediate space becomes open (Fig. 1).

2) Inferior articular cavity:

The inferior articular cavity serves for uniaxial movement of the mandibular 
head as a hinge joint and the axis for this hinge movement is an imaginary line between the medial and lateral poles of the mandibular head. Therefore, the articular surface of the mandibular head moves against the articular surface of the discal fossa, keeping a minute intraarticular space between these articular surfaces. In arthroscopical investigation, this space is designated as the intermediate space in the inferior articular cavity (Fig. 7). Anterior to and posterior to the intermediate space, two intersynovial spaces are present and these are designated as the lower anterior synovial pouch and the lower posterior synovial pouch, respectively. These inter-synovial spaces change their sizes following hinge joint movement of the mandibular head.

3) Articular surface:

The catilaginous articular surfaces usually appear to be smooth, avascular and shinny with ivory-whitish tinge and often reveals streky texture on its surface (Figs. 5, 6, 8). The non-cartilaginous articular surface on the articular disk looks more whitish and compact (Fig. 7) and usually reflects light more brightly than does the aricular cartilage.

4) Synovial membrane:

As compared with the appearance of the articular surfaces, the inner surface of the synovial membrane appears softer and flexible, less shinny with velvet-like texture, and similarly ivory whitish with translucent vascular patterns (Figs. 9, 10, 18). These visual features makes the synovial membrane distinguishable from adjacent articular surfaces (Figs. 8-11). Moreover, the synovial membrane alters its appearance by positional changes of the articular disk following the movement of the lower jaw (Figs. 9, 10).

5) Synovial pouches:

During the movement of the lower jaw, the upper posterior synovial pouch becomes largest among four synovial pouches. The upper posterior synovial pouch begins above from the posterior rim of the cartilaginous articular surface of the mandibular fossa and below from the posterior border of the discal articular surface slightly behind the discal eminence (Fig. 11). The synovial membrane at these upper and lower portions of the upper posterior synovial pouch continues medially, laterally and posteriorly to complete the formation of the synovial pouch (Figs. 11, 12). The retrodiscal fat pad is covered by the synovial membrane of the upper posterior synovial pouch (Figs. 1, 10). Along the both sides of the articular disk at the floor of the upper posterior synovial pouch, bilateral sharrow grooves are formed and continue forward (Fig. 11). Superior to the lateral and medial poles of the mandibular head to which the articular disk are firmly attached, these grooves become deepest (Fig. 13), and thereafter gradually become sharrower again as they continue forward along the flat portion of the articular disk (Fig. 14). These bilateral grooves are designated as the lateral and medial paradiscal synovial grooves.

At varying positions of the articular disk, the synovial membrane often forms inconsistent folds which may become less prominent after alterations of the discal positions (Figs. 9, 10). However, some synovial fold may persist on the surface of the synovial membrane, and are designated as the synovial plica (Fig. 14). Sometimes, one particular type of the synovial plica with tongue-like shape appears. It is so special that it is designated as the tongue-like synovial plica (Figs. 11, 14, 15, 16). In the present investigation, the tongue-like synovial plica was observed at the entrance of the upper posterior synovial pouch behind the medial aspect of the discal eminence in 11 of 20 TMJs. Among them, the 
sizes of the tongue-like synovial plicae were measured in eight TMJs (Table 1). In two other cases, the tongue-like synovial plica was observed in the medial paradiscal synovial groove in the upper posterior synovial pouch (Fig. 14).

The lower posterior synovial pouch is larger than the lower anterior synovial pouch at the opening position of the lower jaw. In the deepest portion of the lower posterior synovial pouch, the anteriorly located condylar surface and the posterioly located synovial surface are facing each other very closely, and form a deep narrow groove without much interspace (Figs. 8, 17). This feature makes arthroscopical view of this pouch differerent from that of the much spacious upper posterior synovial pouch. The synovial membrane of the posterior portion of the lower posterior synovial pouch looks more vascularized due to the blood vesseles of the retrodiscal fat pad present deep to the synovial membrane
(Fig. 18). Moreover, it also shows the configuration revealing the contour of the underlying retrodiscal fat pad (Figs. 8, 18), which is believed to serve as a cushion-like structure for the condylar process of the mandible against the temporal plate when the lower jaw is retracted. Therefore, the synovial membrane in this pouch looks softer and more flexible than that of the upper posterior synovial pouch, and there is no tonguelike synovial plica present.

\section{Discussion}

Arthroscopic observations of the human TMJ have sporadically reported. The earliest report was concerned with the introduction of the arthroscopic technique for the TMJ in patients (Ohnishi, 1975). In the rabbit, arthroscopic findings of the TMJ in normal conditions (Hilsabeck and Laskin, 1978) and in the experimentally induced pathologic conditions (Will-

Table 1. Tongue like synovial plica.

\begin{tabular}{crrcccc}
\hline Case & Age & Sex & $\begin{array}{c}\text { Side of } \\
\text { Joint }\end{array}$ & \multicolumn{4}{c}{ Distance (mm) } \\
\hline 1 & & & a & b & c \\
\hline 2 & 82 & M & L & 3.90 & 1.05 & 21.10 \\
3 & 23 & M & R & 4.75 & 2.00 & 21.00 \\
4 & 89 & M & L & 3.75 & 0.55 & 21.75 \\
5 & 60 & M & L & 6.10 & 0.85 & 17.65 \\
6 & 91 & M & R & 4.45 & 1.40 & 20.55 \\
7 & 77 & M & 6.20 & 2.40 & 20.00 \\
8 & 79 & M & L & 5.65 & 0.75 & 23.00 \\
& & R & 3.40 & 1.85 & 29.07 \\
\hline
\end{tabular}

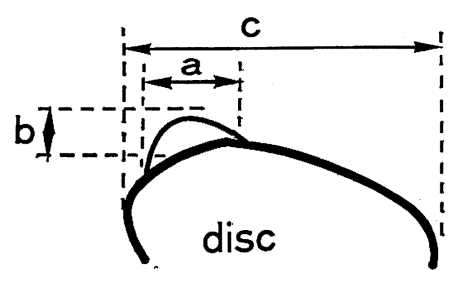


iams and Laskin, 1980) were reported. Recently, using fresh cadavers, the superior articular cavity of the TMJ was observed arthroscopically and macrosscopically, and the synovial membrane and its folds were investigated also microscopically (Kino, 1980; Kino et al., 1981).

In our laboratory, utilizing the preserved cadavers, arthroscopical and gross anatomical investigations of the TMJ have been undertaken (Murakami and Ito, 1980; Murakami and Ono, 1981 ; Murakami and Ito, 1981). For preservation of the cadavers, our improved embalming method (Bradbury and Hoshino, 1977) was used so that the joints were much more movable, even without softening procedures, in comparison with those preserved by conventional embalming methods. However, in order to facilitate easier movements of the specimens, the TMJ and adjacent structures were further softened prior to our investigations.

The appearance of the intra-articular soft tissues such as the synovial membrane and its folds, are usually altered postmortally and by preservation. In situ observations of these features are important clinically and arthroscopically. Therefore, the excised specimens including TMJ are observed macroscopically after immersing in water or arthroscopically after injecting normal saline solution into the articuar cavities. In the present study, arthroscopic findings and gross anatomical features of the TMJ were both taken into account in establishing clinically usable arthroscopical terminology and gross anatomical nomenclature.

The TMJ is unique in the following anatomical aspects.

1) As long as the articular disk is intact, each TMJ consists of two functionally independent joints with their own articular covities, the superior and the inferior articular cavities.
2) The upper surface of the articular disk faces against the concave surface of the mandibular fossa posteriorly and the convex surface of the articular tubercle anteriorly, thus, in sagittal view, it looks like lying-sigmoid shape. The posterior highest point was designated as the discal eminence, and the anterior flat slope as the flat portion of the articular disk to make arthroscopic descrition easier. In the inferior articulra cavity, the surface of the mandibular head is surrounded by the undersurface of the dome-shaped articular disk. This concave space of the articular disk was named as the discal fossa.

3) The articular disk is fused anteriorly and anteromedially with the lateral pterygoid muscle and posteriorly with the retrodiscal fat pad, bilaterally the articular disk is firmly attached to the poles of the mandibular head.

4) The inner surfaces of articular cavities consist of the articular surfaces and the synovial membrane which forms four intersynovial spaces. In this study, intersynovial spaces were designated as the synovial pouches for arthroscopical descriptive purpose.

5) The articular cavities of the TMJ are so narrow that arthroscopically only limited intra-articular features may be observed, although some amounts of normal saline solution are always injected prior to arthroscopy in order to widen the articular cavities. Therefore, gross anatomical subdivisions are not enough to describe arthroscopic findings. Thus, as arthrooscopic terminology the four subdivisions of the superior articular cavity are designated as the upper anterior synovial pouch, the lower intermediate space, the higher intermediate space, and the upper posterior synovial pouch. Similarly, the arthroscopic terminology for three subdivisions of the inferior articular cavity were proposed 
as the lower anterior synovial pouch, the intermediate space, and the lower posterior synovial pouch. Among them, the upper posterior synovial pouch and the lower posterior synovial pouch become larger when the lower jaw is depressed or protruded. When the lower jaw is protruded, the lower intermediate space of the superior articular cavity is closed and the higher intermediate space become open, whereas the latter is closed at the occlusal position.

6) In the upper posterior synovial pouch, largest in size, various synovial folds are formed. Some of these synovial folds were recently described as the postero-discal fold and the oblique protuberance by Kino and his associates (1981). These synovial folds disappear after some discal movements. Therefore in the present study, they were included in a group of non-persistent temporary synovial folds without giving new designation. Some of the synovial folds are, however, distinct and persistent, thus, designated as the synovial plica in the present study. Among them, the tonguelike synovial plica has never been reported previously.

7) The lower posterior synovial pouch is lined by the more flexible and softerlooking synovial membrane with translucent vascular patterns of the underlying retrodiscal fat pad.

8) In the superior articular cavity, the bilateral synovial grooves persist along with the articular disk. Therefore, these were designated as the lateral and medial paradiscal synovial grooves. These grooves seem to be corresponding with the latero-discal and the medio-discal grooves described by Kino et al (1981). Our designation appears to be more precisely describing the characteristics of these synovial grooves.

The purpose of this paper to establish a new series of arthroscopic terminology for the intra-articular features of the TMJ in relation to gross anatomical nomenclature for regional anatomical aspects of the TMJ was fulfiled by presentation of the proposed designations which are anatomically accurate, and at the same arthroscopically meaningful and clinically easy to use.

\section{Acknowledgements}

The authors wish to express our appreciation to our colleagues in Kyoto University, to Dr. Kazutada Ito, Departments of Anatomy and Orthopedic Surgery, for his invaluable assistance in arthroscopic work, and also to Dr. Takatoki Ono, Department of Oral and Maxillofacial Sugery and Dr. Takao Yamamuro, Department of Orthopedic Surgery for their keen interest in our work.

\section{References}

1) Bradbury, S. A. and Hoshino, K.: An improved embalming procedure for long-lasting preservation of the cadaver for anatomical study. Acta anat, 10!: 97-103, 1978.

2) Burman, M.S.: Arthroscopy or the direct visualization of joints. An experimental cadaver study. J Bone and Joint Surg. 13: 669-695, 1931.

3) Hilsabeck, R.B. and Laskin, D.M. : Arthroscopy of the temporomandibular joint of the rabbit. J Oral Surg, 36: 938-943, 1978.

4) Iino, S.: Arthroskopische studien uber die kniegelenke von erwachsenen leichen. J Jap Orthop Surg Soc, 14: 467-518, 1939 (in Japanese).

5) Kino, K.: Morphological and structual observation of the synovial membranes and their folds relating to the endoscopic findings in the upper cavity of the human temporomandibular joint. J Jap Stomatol Soc, $47: 98-134,1980$. (in Japanese).

6) Kino, K., et al.: Morphological observation on the inner surface of the temporo- 
mandibular joint. Histologicl investigation relating to the arthroscopic findings in the upper cavity. Japan J Oral Surg, 27: 1379-1389, 1981 (in Japanese).

7) Murakami, K. and Ito, K.: Arthroscopy of the temporomandibular joint. Abstract of the 6th meeting of the Japanese Arthroscopy Association, p. 1, 1980 (in Japanese).

8) Murakami, K., and Ito, K.: Arthroscopy of the temporomandibular joint. Arthroscopic anatomy and arthroscopic approaches in the human cadaver. Arthroscopy, $6: 1-13,1981$. (in Japanese).

9) Murakami, K. and Ono, T.: Arthroscopic study of the temporomandibular joint. Abstract of the 2nd Gakukansetsu kenkyukai, p. 15, 1981 (in Japanese).

10) Nomina Anatomica. 4th ed., Excerpta Medica, Amsterdam, Oxford, 1977.

11) Ohnishi, M.: Arthroscopy of the temporomandibular joint. J Jap Stomatol Soc, 42 : 207-213, 1975 (in Japanese).

12) Takagi, K.: Arthroskopie. J Jap Orthop. Surg Soc, 14: 359-384, 441-466, 1939. (in Japanese).

13) Watanabe, M., Takeda, S., and Ikeuchi, H. : Atlas of arthroscopy. Igaku shoin, Tokyo, 1969.

14) Williams, R.A., and Laskin, D.M. : Arthroscpic examination of experimentally induced pathologic conditions of the rabbit temporomandibular joint. J Oral Surg, 38: 652-659, 1980. 

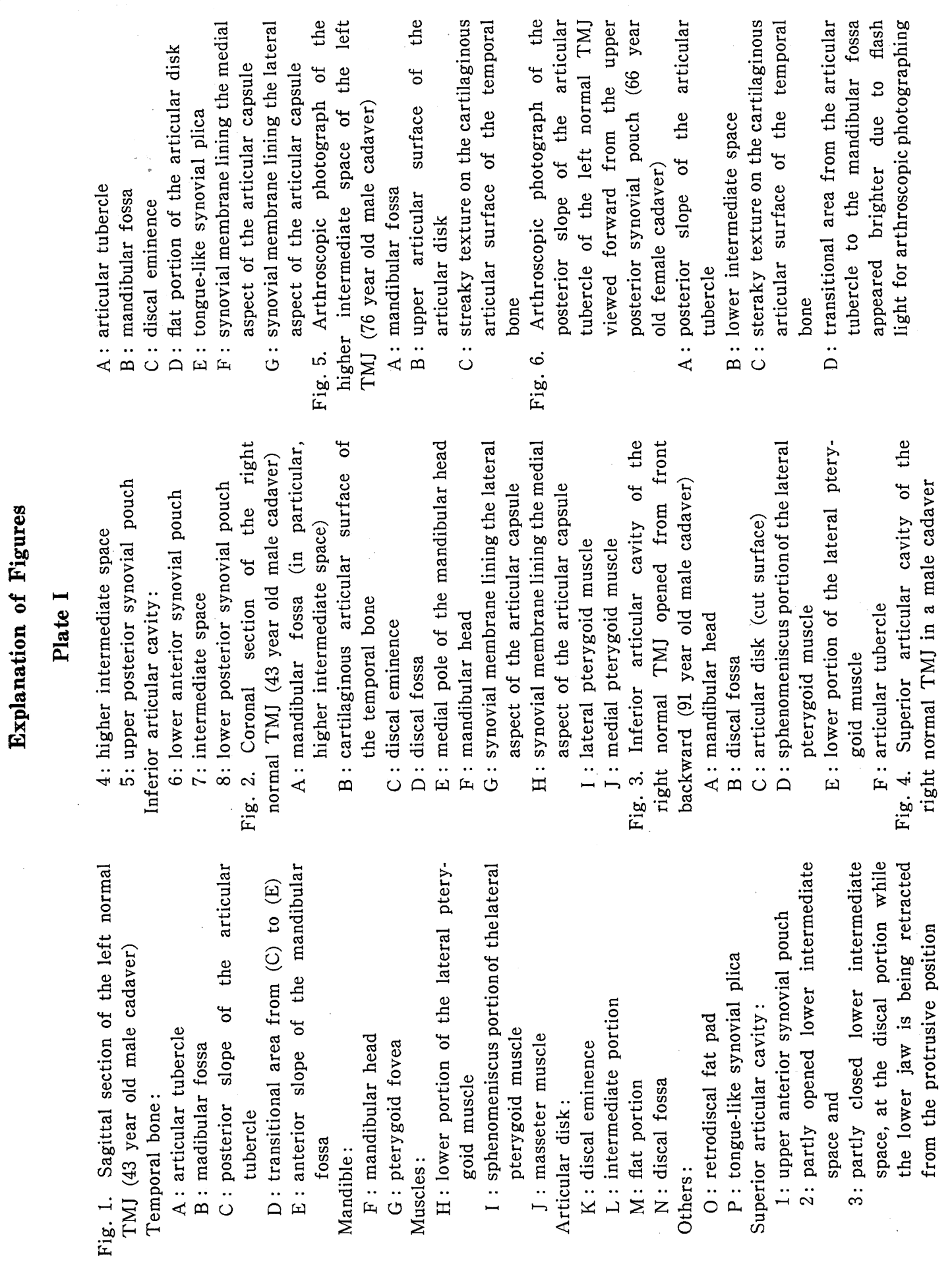
Plate I
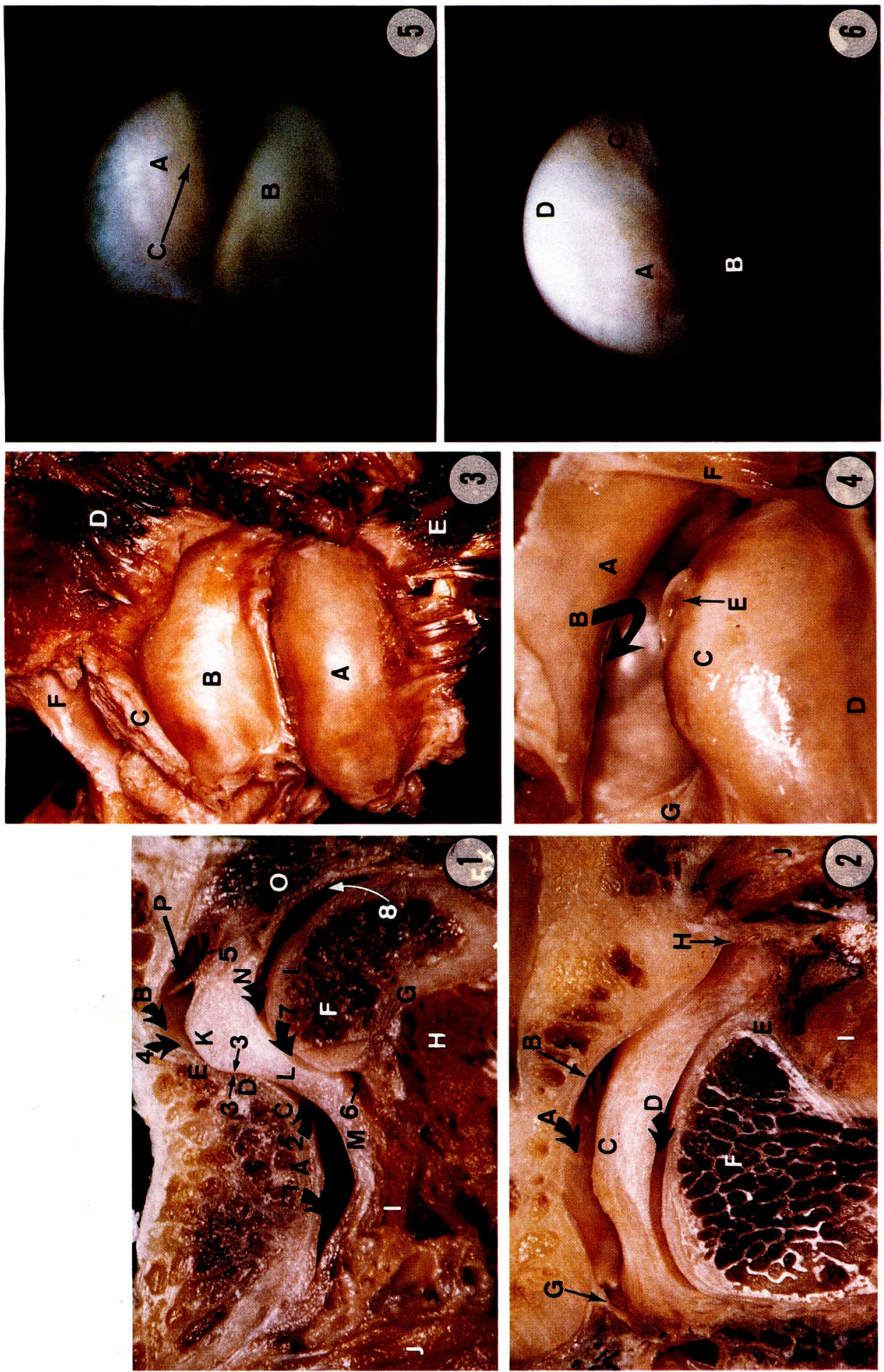

K. Murakami and K. Hoshino 

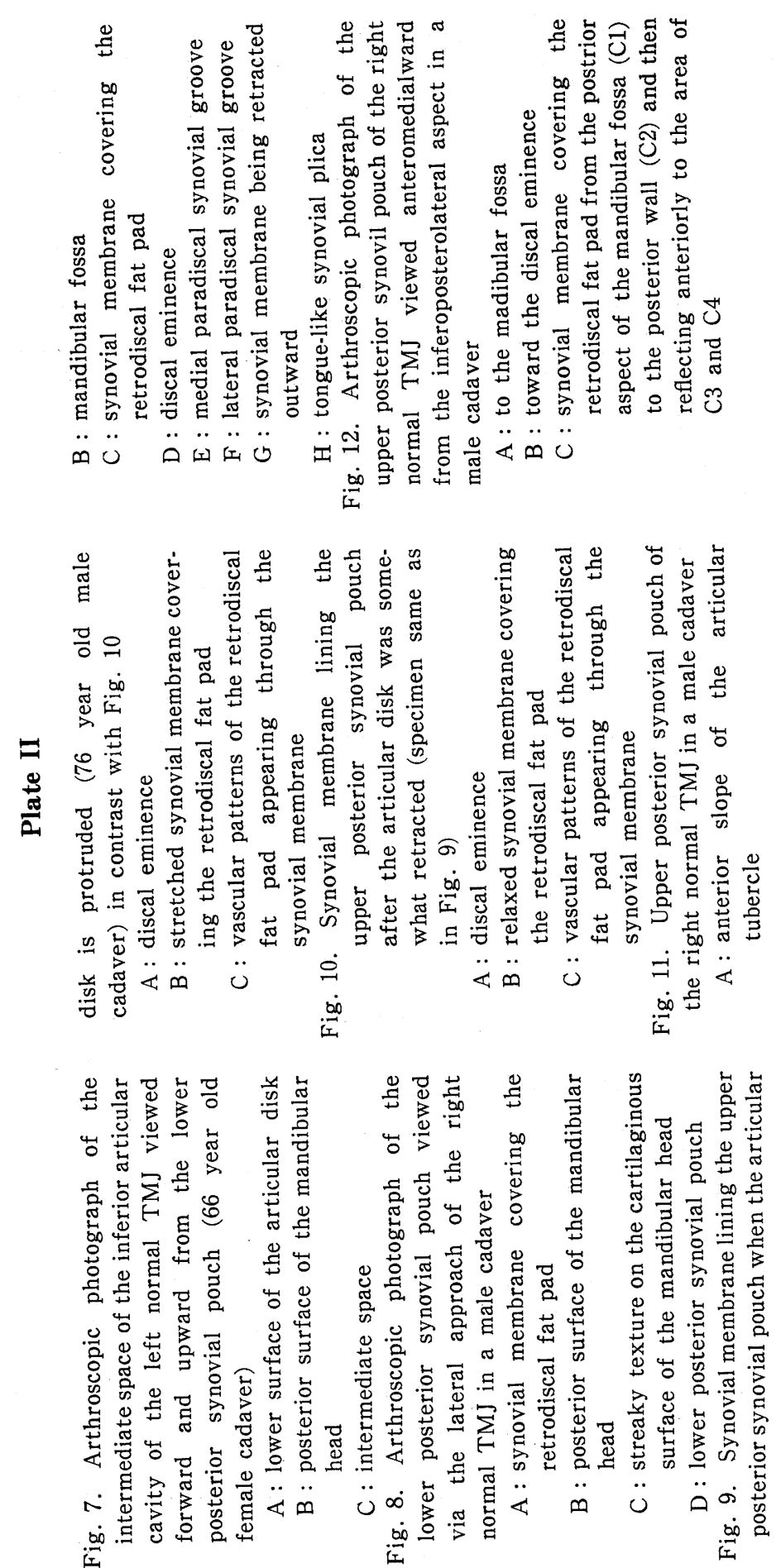
Plate II
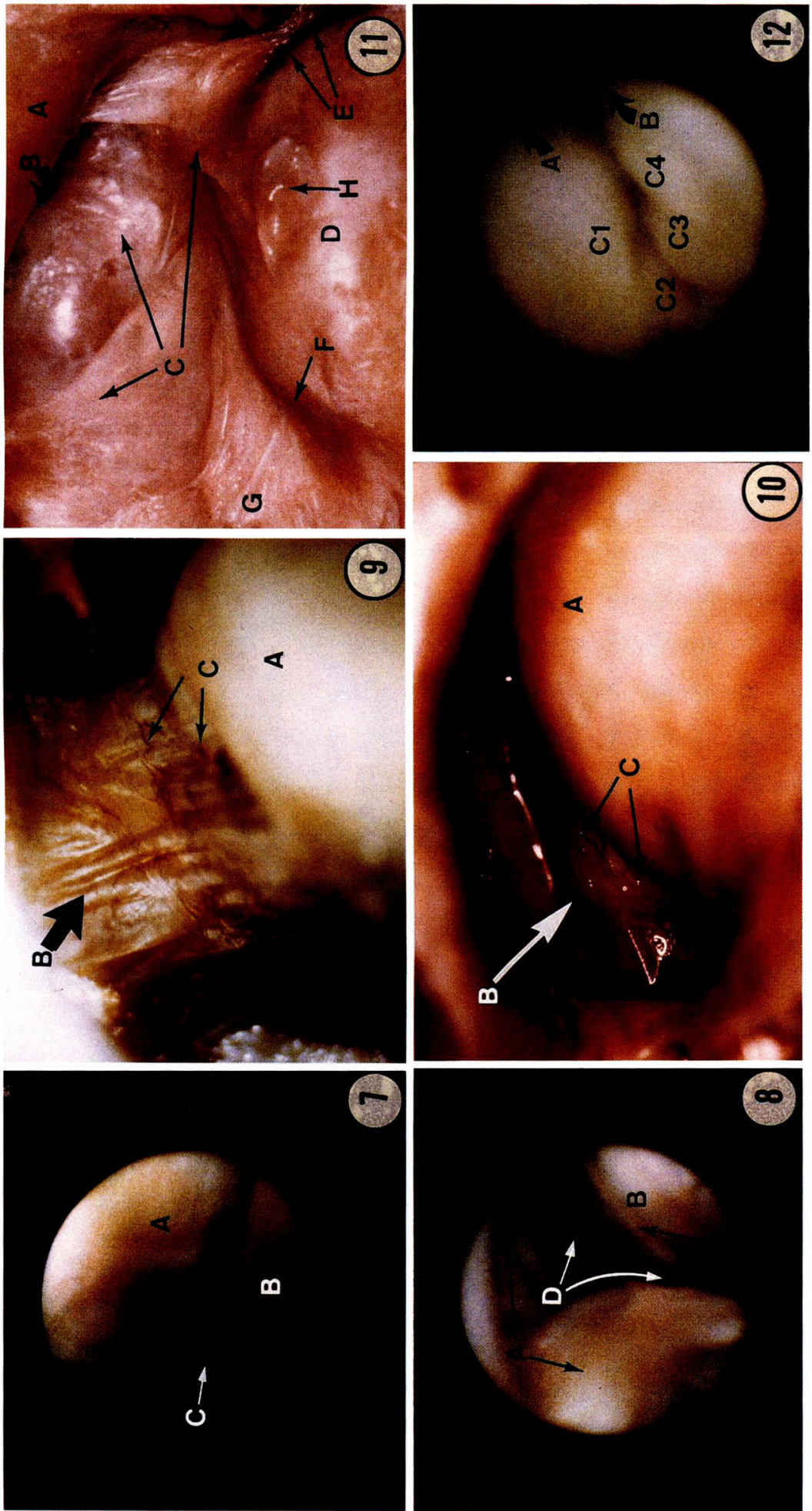

(

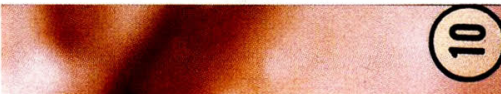

os
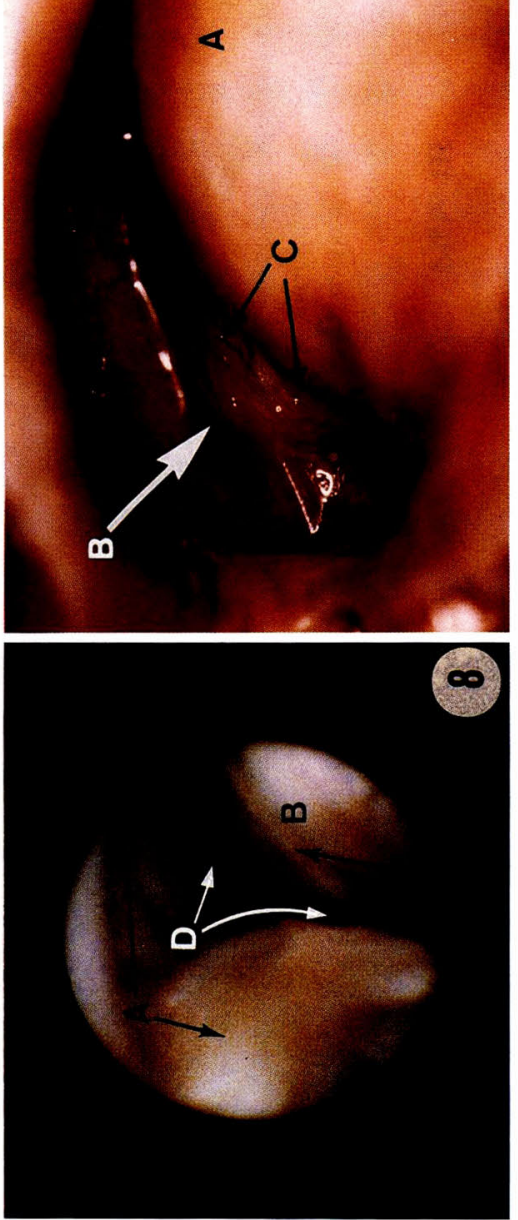

K. Murakami and K. Hoshino 

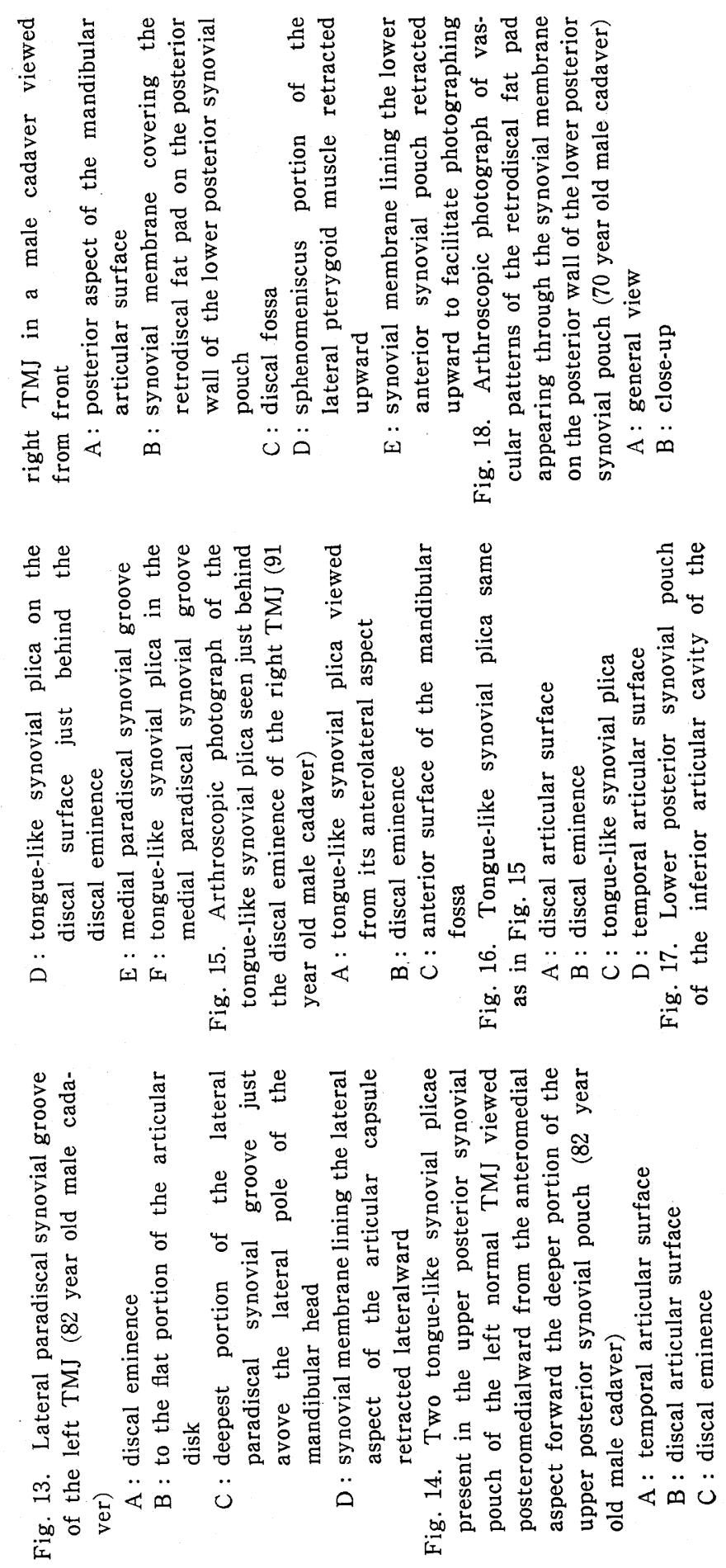

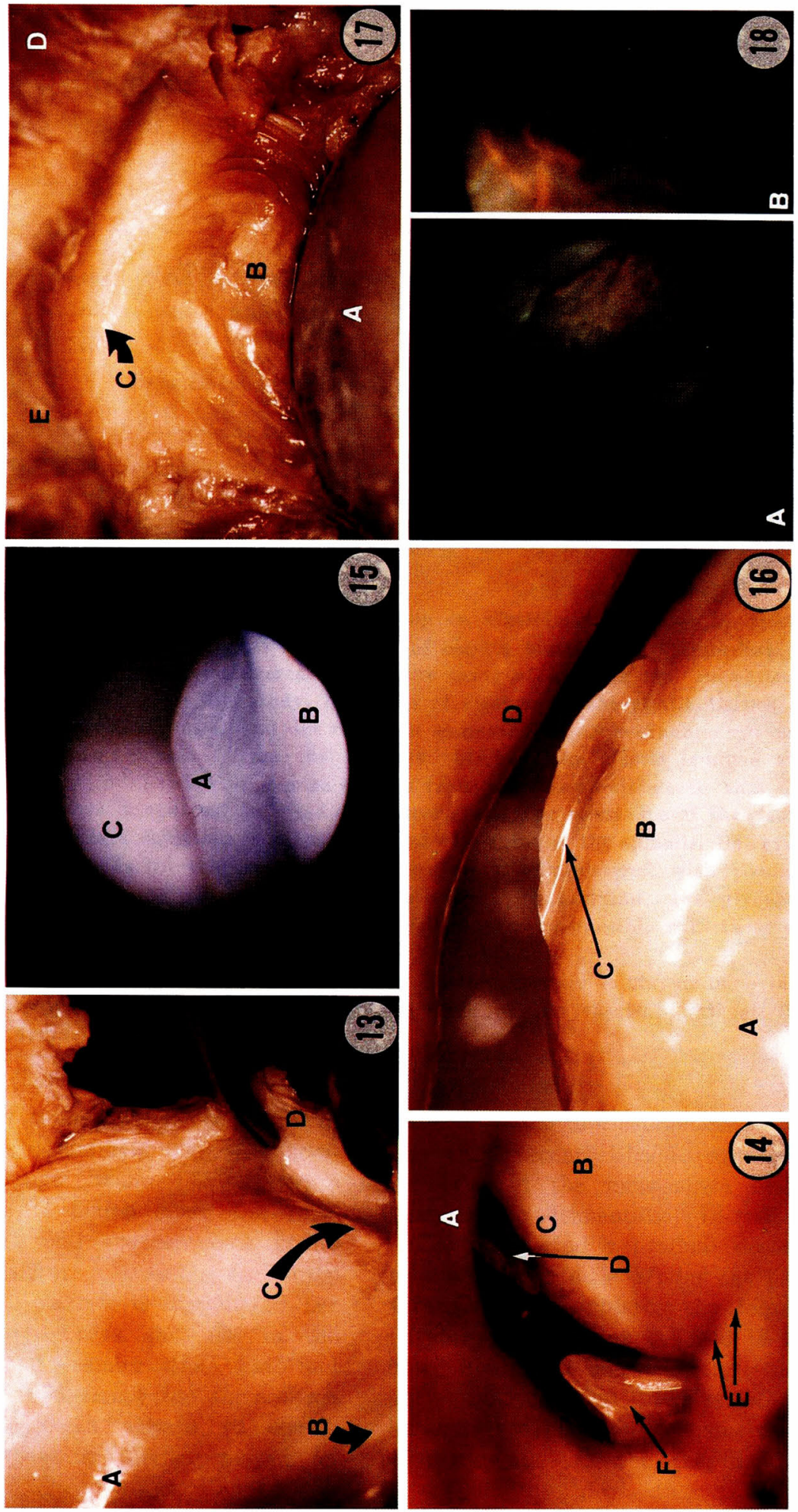

(9)
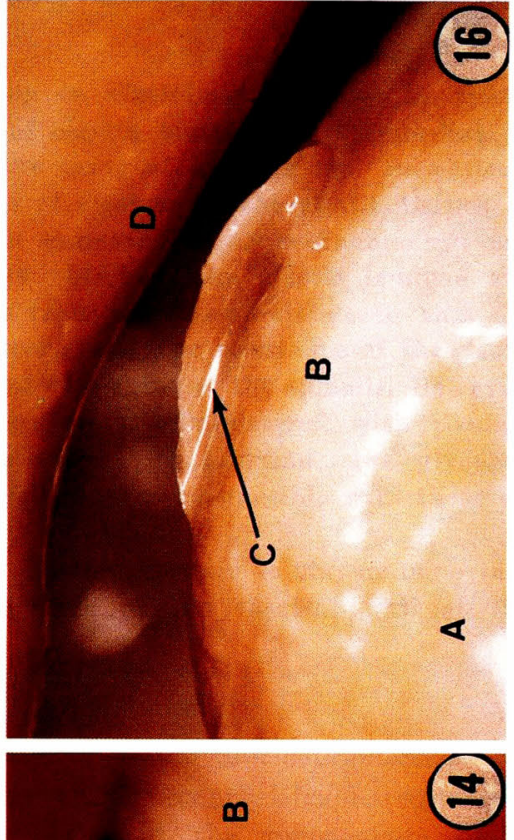

K. Murakami and K. Hoshino

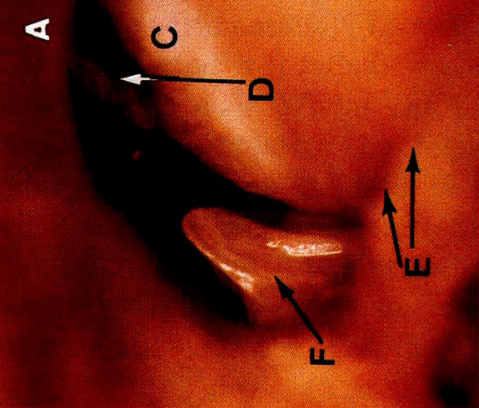

